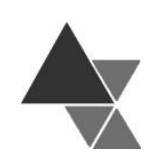

\title{
Contribuição da alimentação fora do domicílio para a ingestão de nutrientes antioxidantes no Brasil
}

\author{
Luciana Zabine de Oliveira ${ }^{1}$, Camila Tureck ${ }^{2}$ e Eloá Angélica Koehnlein ${ }^{3}$
}

Tem sido observado no Brasil o aumento das refeições realizadas fora do domićlío e a importância dos antioxidantes da dieta para a prevenção das doenças crônicas não transmissíveis. O objetivo do trabalho foi avaliar a contribuição da alimentação fora do domicílio para a ingestão de nutrientes antioxidantes da população brasileira. Foram utilizados os dados do registro alimentar de um dia de 34.003 brasileiros, descritos na Pesquisa de Orçamentos Familiares 2008-2009. Avaliou-se a ingestão de vitaminas A, C e E, e minerais zinco, selênio, cobre e manganês, sendo utilizadas tabelas de composição de alimentos nacionais e internacional. Os dados foram organizados e analisados pelo software Microsoft Excel ${ }^{\circledR} 2010$ e programa Bioestat ${ }^{\circledR}$ versão 5.3. A participação das

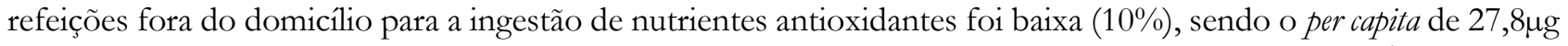
para vitamina $A, 8,97 \mathrm{mg}$ para vitamina $C, 0,57 \mathrm{mg}$ para vitamina $\mathrm{E}, 1,37 \mathrm{mg}$ para zinco, $10,13 \mu \mathrm{g}$ para selênio, $0,15 \mathrm{mg}$ para cobre e $0,32 \mathrm{mg}$ para manganês. Os grupos alimentares que mais contribuíram para a ingestão de nutrientes antioxidantes fora do domicilio foram as frutas, legumes e verduras, carnes e ovos, leguminosas e cereais. Devido ao crescimento das refeições fora do domicilio é fundamental incentivar à ingestão de nutrientes antioxidantes, por meio desse tipo de refeição.

Palavras-chave: consumo alimentar, alimentação fora do domicílio, antioxidantes, vitaminas, minerais.

\section{Contribution of eating outside the home to the intake of antioxidant nutrients in Brazil}

Has been observed in Brazil the increase in outside home meals and the importance of dietary antioxidants in the prevention of chronic non-transmitted diseases. The objective of the study was to evaluate the contribution of outside home meals for the intake antioxidant nutrients of the Brazilian population. Were utilized the data of one day food records of 34.003 people, which was described the Family Budget Survey 2008-2009. It was evaluated the ingestion of vitamins $\mathrm{A}, \mathrm{C}$ and $\mathrm{E}$, and minerals zinc, selenium, copper and manganese, utilizing national and international tables of food composition. The data were analysed and organized with the software Microsoft Excel ${ }^{\circledR} 2010$ and Bioestat ${ }^{\circledR}$ program version 5.3. The participation of outside home meals to the intake antioxidant nutrients was low (10\%), and the per capita: $27,8 \mu \mathrm{g}$ to vitamin $\mathrm{A}, 8,97 \mathrm{mg}$ to vitamin $\mathrm{C}, 0,57 \mathrm{mg}$ to vitamin $\mathrm{E}$,

${ }^{1}$ Pós-Graduada em Segurança Alimentar e Nutricional - UFFS, Campus de Realeza, PR.

2 Pós-Graduada em Residência Multiprofissional em Neurologia. Universidade da Região de Joinville - Univille, Joinville, SC.

${ }^{3}$ Docente do Curso de Graduação em Nutrição e do Programa de Pós-Graduação em Ciência e Tecnologia de Alimentos - Universidade Federal da Fronteira Sul, Campus Realeza - PR. Endereço para correspondência: Rua Edmundo Gaiaevisk no 1.000, Zona Rural, Realeza, Paraná, PR. E-mail: eloa.koehnlein@uffs.edu.br 
1,37 $\mathrm{mg}$ to zinc, $10,13 \mu \mathrm{g}$ to selenium, $0,15 \mathrm{mg}$ to cooper and $0,32 \mathrm{mg}$ to manganese. The food groups that most contributed to the ingestion of antioxidant nutrients in outside home were fruits and vegetables, meats and eggs, legumes and cereals. Due to the growth of meals outside the home is essential to encourage the intake of antioxidant nutrients, through this type of meal.

Keywords: food consumption, eating outside the home, antioxidant, vitamins, minerals.

\section{INTRODUÇÃO}

Um dos temas mais importantes para a área de segurança alimentar e nutricional são as mudanças no consumo alimentar, observadas em praticamente a totalidade dos países. A urbanização implicou em uma mudança de tradição, já que com a inserção da mulher no mercado de trabalho houve redução no tempo de preparo das refeições. Assim, o comércio e indústria alimentícia oferecem, cada vez mais, alimentos práticos, duráveis, palatáveis e atrativos para as pessoas $[1,2,3]$.

Além do aumento do consumo de alimentos industrializados, observa-se também um crescimento no consumo de alimentos fora do domicílio. Segundo Bezerra et al. (2013) [4], os fatores sociais, demográficos e econômicos, bem como a maior participação da mulher no mercado de trabalho, o aumento da renda familiar, a urbanização, a escassez de tempo da sociedade moderna e a diminuição do preço de alimentos prontos para consumo, influenciaram o aumento nos gastos com a alimentação fora do domicílio.

Assim, verificou-se que a participação da alimentação fora do domicílio teve aumento de $25 \%$, quando são comparados os gastos com aquisições de alimentos e bebidas para consumo fora do domicílio na Pesquisa de Orçamentos Familiares (POF) realizada em 2002/2003 e 2008/2009[5]. Ainda, de acordo com os dados da POF 2008-2009, a despesa com alimentação fora do domicílio foi de $31,1 \%$, sendo maior na área urbana $(33,1 \%)$, em relação à rural $(17,5 \%)$. Em comparação à pesquisa realizada em 2002/2003, observou-se um aumento de sete pontos percentuais no peso da despesa com alimentação fora do domicílio[ ${ }^{[\theta]}$.

A principal consequência da mudança nos hábitos alimentares da população brasileira é a transição nutricional que vêm sendo observada desde o século passado, sendo caracterizada pela redução na prevalência de desnutrição e aumento na prevalência de obesidade. A obesidade está relacionada com o surgimento de diversas comorbidades, tais como dislipidemias, doenças cardiovasculares e diabetes mellitus que estão associadas ao aumento do consumo de alimentos com elevada densidade energética, ricos em açúcares, gorduras e sódio e pobres em micronutrientes importantes ${ }^{[1]}$.

Segundo Catania, Barros e Ferreira (2009)[7], na cadeia respiratória ocorre uma sequência de reações geradoras de eletronegatividade, onde são gerados naturalmente ou por alguma disfunção biológica, os radicais livres. O estresse oxidativo surge quando há um desequilíbrio no estado de óxido-redução, em favor das reações pró-oxidativas, causando danos ao organismo.

Conforme pesquisa realizada por Gottlieb, Morassutti e Cruz (2011)[8], o estresse oxidativo está na base das doenças crônicas não transmissíveis, principalmente doenças cardiovasculares, diabetes tipo 2 e câncer. Isso pelo fato de causar disfunção endotelial e mitocondrial, aumento do processo inflamatório e alteração no padrão de metilação do DNA.

Nos mecanismos de defesa para prevenir o estresse oxidativo participam as enzimas endógenas (superóxido-dismutase, catalase e glutationaperoxidase ${ }^{[7]}$. No entanto, acredita-se que as mesmas não são infalíveis, e assim se faz importante a ingestão de antioxidantes provenientes da dieta, dentre eles estão as vitaminas $\mathrm{A}, \mathrm{C}$ e E e os minerais zinco, selênio, cobre e manganês ${ }^{[?]}$.

A vitamina A e os carotenoides têm capacidade de reagir em baixas concentrações e de modular o metabolismo do carcinoma, inibindo a proliferação celular ${ }^{[10,7]}$. A vitamina E, especificamente a fração $\alpha$-tocoferol, é uma das mais estudadas, e tratase da principal vitamina antioxidante presente no plasma e na partícula de lipoproteína de baixa densidade (LDL) [11,12,13,14]. O ácido ascórbico (vitamina C) é um 
importante antioxidante do meio aquoso e possui a função de reciclar a vitamina $\mathrm{E}[14,15,16]$.

Em relação aos minerais observa-se que estes estão envolvidos em várias reações antioxidantes indiretamente, por atuarem como cofatores enzimáticos da defesa antioxidante endógena. $\mathrm{O}$ zinco e o cobre são importantes componentes da enzima superóxido-dismutase. O selênio atua como cofator da enzima glutationa-peroxidase, e tem relevante importância clínica na prevenção e tratamento do diabetes tipo 2 e das doenças cardiovasculares. Já o manganês é o componente de várias enzimas inclusive superóxido-dismutase mitocondrial [7,14,17,18].

Assim, observando-se as mudanças no padrão alimentar dos brasileiros, bem como o crescimento das refeições realizadas fora do domicílio e das doenças crônicas não transmissíveis, e considerando-se a importância do consumo de antioxidantes, esse estudo teve como objetivo avaliar a participação da alimentação fora do domicílio para a ingestão de vitaminas e minerais antioxidantes na dieta brasileira.

\section{MÉTODOS}

Para a estimativa do consumo de alimentos e bebidas dentro e fora do domicílio foram utilizados os dados do Inquérito Nacional de Alimentação (INA), que constituiu um módulo da Pesquisa de Orçamentos Familiares (POF) 2008-2009. Na POF (2008-2009), a mais recente disponível, realizou-se pela primeira vez uma pesquisa sobre a ingestão alimentar em uma sub amostra de 13.569 domicílios selecionados aleatoriamente, o que correspondeu a 24,3\% dos 55.970 domicílios investigados.

Foram coletados dados do consumo alimentar de 34.003 indivíduos com dez anos ou mais de idade, de todas as regiões geográficas do país, residentes na zona urbana e rural. $\mathrm{O}$ consumo alimentar foi registrado em dois dias não consecutivos, considerando o tipo de preparação, a medida caseira usada, a quantidade consumida, o horário de consumo, e se o mesmo ocorreu no domicílio ou fora do domicílio. No entanto, a POF (2008-2009) considerou o primeiro dia de registro para a estimativa do consumo alimentar médio dos indivíduos, devido a qualidade da informação obtida $\left.{ }^{[}\right]$.
O método utilizado nesta pesquisa foi semelhante à utilizada por Tureck et al. (2013)[19], na qual os alimentos relatados pelos participantes foram distribuídos em quinze grupos com base na POF (20082009), os quais foram inseridos em um banco de dados, totalizando 188 itens alimentares. Os grupos estudados foram: 1. Cereais; 2. Leguminosas; 3. Verduras e legumes; 4. Raízes e tubérculos; 5. Frutas; 6. Oleaginosas; 7. Farinhas, massas, panificados e biscoitos; 8. Carnes e ovos; 9. Laticínios; 10. Doces; 11. Óleos e gorduras; 12. Bebidas; 13. Salgados e sanduíches; 14. Sopas e caldos; 15. Molhos e condimentos.

O número de grupos alimentares constituiu naqueles que a POF avaliou, sendo incluídos em um mesmo grupo aqueles que se assemelhavam (como verduras e legumes, por exemplo). $\mathrm{O}$ número total de itens alimentares resultou da exclusão dos alimentos que contém várias denominações para um mesmo alimento (como tangerina, ponkan, mexerica, por exemplo), bem como da exclusão dos alimentos light e diet, visto que a maioria das tabelas de composição de alimentos considera as informações contidas no rótulo desses produtos, o qual não contém os micronutrientes que foram alvos de interesse do presente trabalho. Ainda, foram excluídos os alimentos descritos como orgânicos, uma vez que as tabelas de composição não apresentam informações específicas para estes alimentos.

A seleção e a caracterização foram realizadas com base no modo de preparo habitual de consumo, ou seja, considerou-se o alimento na forma crua, cozida, assada, refogada ou frita. Verduras folhosas e frutas foram consideradas na forma crua, já os legumes na forma cozida. As carnes, ovos, leguminosas, raízes e tubérculos, e alguns cereais como o arroz, também foram considerados na forma cozida. Os demais alimentos e preparações foram considerados conforme se encontram disponíveis para alimentação.

As categorias da POF (2008-2009) descritas como "preparações à base de" foram quantificadas considerando-se o alimento principal. Já para categorias denominadas "outras" considerou-se os alimentos com maior prevalência de consumo.

Para a quantificação dos teores das vitaminas e minerais antioxidantes dos alimentos selecionados foram utilizadas as tabelas de composição de alimentos 
nacionais e internacional[20,21,22,23]. Para cada alimento os dados de pelo menos duas referências foram adotados, a fim de possibilitar o cálculo do teor médio, sendo que uma das informações preferencialmente foi obtida da Tabela Brasileira de Composição dos Alimentos ${ }^{[20]}$. Os teores das vitaminas e minerais para cada $100 \mathrm{~g}$ de alimentos foram convertidos de acordo com a porção média de alimentos per capita consumida no domicílio e fora do domicílio, a partir da proporção (\%) consumida em relação ao total. Destaca-se que para a vitamina $A$ foram obtidos os valores de retinol ou equivalentes de atividade de retinol (RAE).

A ingestão total de cada micronutriente antioxidante foi calculada a partir do consumo alimentar médio per capita diário, considerando as seguintes variáveis: sexo (feminino e masculino), situação de domicílio (zona rural e urbana), Brasil e grandes regiões (Norte, Nordeste, Sul, Sudeste, CentroOeste), estágios de vida (adolescentes (10 a 18 anos), adultos (19 a 59 anos) e idosos (>60 anos)), classes de rendimento (até $\mathrm{R} \$ 296$, mais de $\mathrm{R} \$ 296$ a $\mathrm{R} \$ 571$, mais de $R \$ 571$ a $R \$ 1.089$, mais de $R \$ 1.089$, e consumo dentro e fora do domicílio.
Os dados foram organizados e analisados por meio do software Microsoft Exce ${ }^{\mathbb{B} 2010}$ e do programa Bioestat ${ }^{\circledR}$ versão 5.3 , sendo expressos como média, desvio padrão e percentual de contribuição dos grupos alimentares e dos alimentos em relação à ingestão total dos antioxidantes considerados no estudo.

As comparações entre os dados obtidos foram realizadas utilizando-se o teste $t$-student, considerando-se $5 \%$ de significância.

\section{RESULTADOS E DISCUSSÃO}

A análise comparativa da ingestão de vitaminas com ação antioxidante dentro e fora do domicílio demonstrou que a alimentação fora do domicílio contribuiu em média com $9,8 \%$ da ingestão total de vitaminas antioxidantes. Conforme os dados apresentados na Tabela 1, para todas as vitaminas a ingestão foi significativamente superior $(p<0,05)$ no domicílio em relação à ingestão fora deste.

Tabela 1. Estimativa da ingestão de vitaminas antioxidantes pela população brasileira no domicílio e fora do domicílio de acordo com diferentes variáveis. Brasil, 2008-2009

\begin{tabular}{|c|c|c|c|c|c|c|c|c|c|c|c|c|c|c|c|}
\hline & \multicolumn{5}{|c|}{ Vitamina A ( $\mu \mathrm{g} /$ dia $)$} & \multicolumn{5}{|c|}{ Vitamina C (mg/dia) } & \multicolumn{5}{|c|}{ Vitamina E (mg/dia) } \\
\hline & \multicolumn{2}{|c|}{ D } & \multicolumn{2}{|c|}{ FD } & \multirow{2}{*}{$\begin{array}{r}\text { \%FD } \\
9,00\end{array}$} & \multicolumn{2}{|c|}{ D } & \multicolumn{2}{|c|}{ FD } & \multirow{2}{*}{$\begin{array}{r}\% \text { FD } \\
9,66\end{array}$} & \multicolumn{2}{|c|}{$\mathbf{D}$} & \multicolumn{2}{|c|}{ FD } & \multirow{2}{*}{$\begin{array}{r}\text { \%FD } \\
10,71\end{array}$} \\
\hline População total & 281,14 & $(6,17)$ & 27,80 & $(0,09)^{*}$ & & 83,87 & $(6,91)$ & 8,97 & $(0,64)^{*}$ & & 4,75 & $(0,93)$ & 0,57 & $(0,05)^{*}$ & \\
\hline \multicolumn{16}{|l|}{ Sexo } \\
\hline Sexo Masculino & 281,00 & $(12,20)$ & 56,00 & $(3,40)^{*}$ & 16,62 & 73,29 & $(6,70)$ & 18,40 & $(1,63)^{*}$ & 20,07 & 4,86 & $(0,87)$ & 0,99 & $(0,15)^{*}$ & 16,92 \\
\hline Sexo Feminino & 282,20 & $(4,30)$ & 39,20 & $(1,40)^{*}$ & 12,20 & 80,25 & $(5,45)$ & 13,65 & $(1,38)^{*}$ & 14,54 & 4,22 & $(0,85)$ & 0,63 & $(0,11)^{*}$ & 12,99 \\
\hline \multicolumn{16}{|c|}{ Situação do domicilio } \\
\hline Urbano & 278,49 & $(10,19)$ & 49,05 & $(3,75)^{*}$ & 14,98 & 76,96 & $(6,90)$ & 16,07 & $(1,89)^{*}$ & 17,27 & 4,46 & $(0,91)$ & 0,82 & $(0,13)^{*}$ & 15,53 \\
\hline Rural & 317,31 & $(1,70)$ & 30,57 & $(5,26)^{*}$ & 8,79 & 79,55 & $(1,95)$ & 13,69 & $(0,82)^{*}$ & 14,68 & 5,12 & $(0,68)$ & 0,49 & $(0,06)^{*}$ & 8,73 \\
\hline \multicolumn{16}{|l|}{ Região } \\
\hline Norte & 260,23 & $(16,05)$ & 49,24 & $(2,51)^{*}$ & 15,91 & 73,94 & $(5,40)$ & 20,48 & $(1,03)^{*}$ & 21,69 & 4,79 & $(0,67)$ & 0,85 & $(0,08)^{*}$ & 15,07 \\
\hline Nordeste & 344,74 & $(19,18)$ & 46,68 & $(1,99)^{*}$ & 11,93 & 68,11 & $(5,65)$ & 14,80 & $(0,69)^{*}$ & 17,85 & 4,74 & $(5,39)$ & 0,64 & $(0,09)^{*}$ & 11,90 \\
\hline Sudeste & 246,90 & $(3,11)$ & 42,19 & $(2,94)^{*}$ & 14,59 & 78,84 & $(6,61)$ & 14,80 & $(1,84)^{*}$ & 15,81 & 4,49 & $(0,95)$ & 0,85 & $(0,15)^{*}$ & 15,92 \\
\hline Sul & 297,90 & $(1,71)$ & 55,30 & $(2,54)^{*}$ & 15,66 & 91,80 & $(6,32)$ & 17,27 & $(2,00)^{*}$ & 15.83 & 4,25 & $(0,98)$ & 0,81 & $(0,12)^{*}$ & 16,01 \\
\hline Centro-Oeste & 254,49 & $(5,19)$ & 46,80 & $(1,01)^{*}$ & 15,53 & 74,21 & $(4,74)$ & 18,10 & $(1,84)^{*}$ & 19,61 & 4,85 & $(0,74)$ & 0,88 & $(0,16)^{*}$ & 15,36 \\
\hline \multicolumn{16}{|l|}{ Estágio de vida } \\
\hline Adolescentes & 270,57 & $(14,50)$ & 38,67 & $(4,43)^{*}$ & 12,50 & 72,98 & $(8,82)$ & 13,43 & $(1,42)^{*}$ & 15,54 & 4,80 & $\pm 0,87$ & 0,64 & $(0,06)^{*}$ & 11,76 \\
\hline Adultos & 264,11 & $(5,94)$ & 66,38 & $(3,72)^{*}$ & 20,09 & 74,99 & $(5,84)$ & 17,95 & $(1,66)^{*}$ & 19,31 & 4,53 & $\pm 0,90$ & 0,88 & $(0,11)^{*}$ & 16,27 \\
\hline Idosos & 331,13 & $(0,50)$ & 15,52 & $(1,64)^{*}$ & 4,48 & 95,28 & $(3,31)$ & 6,87 & $(0,69)^{*}$ & 6,73 & 4,54 & $\pm 0,89$ & 0,26 & $(0,05)^{*}$ & 5,42 \\
\hline \multicolumn{16}{|c|}{ Classes de rendimento } \\
\hline $\begin{array}{l}\text { Renda até } \\
\mathrm{R} \$ 296,00\end{array}$ & 267,41 & $(13,33)$ & 30,57 & $(1,29)^{*}$ & 10,27 & 56,63 & $(5,07)$ & 12,14 & $(0,80)^{*}$ & 17,65 & 4,67 & $(0,75)$ & 0,54 & $(0,06)^{*}$ & 10,36 \\
\hline $\begin{array}{lr}\text { Renda > } \\
\mathrm{R} \$ 296,00\end{array}$ & 271,23 & $(12,55)$ & 44,55 & $(1,38)^{*}$ & 14,10 & 69,25 & $(14,41)$ & 14,41 & $(1,02)^{*}$ & 17,22 & 4,71 & $(0,93)$ & 0,69 & $(0,11)^{*}$ & 12,78 \\
\hline $\begin{array}{l}\mathrm{R} \$ 571,00 \\
\text { Renda > que } \\
\mathrm{R} \$ 571,00 \quad \text { a }\end{array}$ & 270,39 & $(6,70)$ & 46,56 & $(3,14)^{*}$ & 14,70 & 83,48 & $(7,32)$ & 16,28 & $(1,76)^{*}$ & 16,32 & 4,48 & $(0,91)$ & 0,83 & $(0,13)^{*}$ & 15,63 \\
\hline $\begin{array}{l}\mathrm{R} \$ 1.089,00 \\
\mathrm{Renda}> \\
\mathrm{R} \$ 1.089,00\end{array}$ & 332,15 & $(1,87)$ & 79,18 & $(5,43)^{*}$ & 19,26 & 105,29 & $(5,92)$ & 21,67 & $(2,58)^{*}$ & 17,07 & 4,28 & $\pm(0,92)$ & 1,16 & $(0,20)^{*}$ & 21,32 \\
\hline
\end{tabular}


Quanto à ingestão de minerais antioxidantes, demonstrado nas Tabelas 2 e 3, observou-se que a alimentação fora do domicílio contribuiu em média com $10,8 \%$ da ingestão total, assemelhando-se à contribuição na ingestão de vitaminas antioxidantes.
Ainda, observou-se que a ingestão de minerais antioxidantes também foi significativamente superior no domicílio $(p<0,05)$, quando comparada à ingestão fora do mesmo.

Tabela 2. Estimativa da ingestão dos minerais antioxidantes zinco e selênio da população brasileira no domicílio e fora do domicilio de acordo com diferentes variáveis. Brasil 2008-2009

\begin{tabular}{|c|c|c|c|c|c|c|c|c|c|c|}
\hline & \multicolumn{5}{|c|}{ Zinco (mg/dia) } & \multicolumn{5}{|c|}{ Selênio ( $\mu \mathrm{g} / \mathrm{dia})$} \\
\hline & \multicolumn{2}{|c|}{$\mathbf{D}$} & \multicolumn{2}{|c|}{ FD } & \multirow{2}{*}{$\begin{array}{l}\mathbf{0 F D} \\
11,66\end{array}$} & \multicolumn{2}{|c|}{$\mathbf{D}$} & \multicolumn{2}{|c|}{ FD } & \multirow{2}{*}{$\begin{array}{r}\mathbf{0 F D} \\
9,41\end{array}$} \\
\hline Populacão total & 10,38 & $(0,81)$ & 1,37 & $(0,16)^{*}$ & & 97,52 & $(2,06)$ & 10,13 & $(0,71)^{*}$ & \\
\hline \multicolumn{11}{|l|}{ Sexo } \\
\hline Sexo Masculino & 10,83 & $(0,79)$ & 2,34 & $(0,14)^{*}$ & 17,08 & 100,25 & $(2,75)$ & 19,94 & $(0,75)^{*}$ & 16,59 \\
\hline Sexo Feminino & 8,85 & $(0,62)$ & 1,35 & $(0,09)^{*}$ & 13,24 & 83,22 & $(2,01)$ & 12,66 & $(0,61)^{*}$ & 13,20 \\
\hline \multicolumn{11}{|c|}{ Situação do domicílio } \\
\hline Urbano & 9,51 & $(0,68)$ & 1,93 & $(0,12)^{*}$ & 16,87 & 88,09 & $(2,01)$ & 16,91 & $(0,84)^{*}$ & 16,10 \\
\hline Rural & 11,26 & $(0,94)$ & 1,13 & $(0,05)^{*}$ & 9,12 & 109,49 & $(4,16)$ & 11,04 & $(0,29)^{*}$ & 9,16 \\
\hline \multicolumn{11}{|l|}{ Região } \\
\hline Norte & 10,26 & $(0,39)$ & 1,70 & $(0,07)^{*}$ & 14,21 & 128,46 & $(4,00)$ & 19,91 & $(1,02)^{*}$ & 13,42 \\
\hline Nordeste & 10,19 & $(0,74)$ & 1,33 & $(0,08)^{*}$ & 11,55 & 99,00 & $(0,60)$ & 13,12 & $(0,40)^{*}$ & 11,70 \\
\hline Sudeste & 9,44 & $(0,65)$ & 1,82 & $(0,18)^{*}$ & 16,16 & 83,34 & $(2,93)$ & 16,10 & $(0,83)^{*}$ & 16,19 \\
\hline Sul & 10,03 & $(1,02)$ & 1,94 & $(0,16)^{*}$ & 16,21 & 87,34 & $(2,18)$ & 17,29 & $(0,85)^{*}$ & 16,52 \\
\hline Centro-Oeste & 10,58 & $(0,63)$ & 2,03 & $(0,15)^{*}$ & 16,10 & 85,87 & $(3,68)$ & 15,20 & $(0,79)^{*}$ & 15,04 \\
\hline \multicolumn{11}{|l|}{ Estágio de vida } \\
\hline Adolescentes & 10,14 & $(0,75)$ & 1,33 & $(0,09)^{*}$ & 11,60 & 94,36 & $(2,38)$ & 13,29 & $(0,69)^{*}$ & 12,35 \\
\hline Adultos & 9,65 & $(0,70)$ & 2,24 & $(0,14)^{*}$ & 18,84 & 90,86 & $(2,21)$ & 19,37 & $(1,02)^{*}$ & 17,57 \\
\hline Idosos & 9,70 & $(0,65)$ & 0,60 & $(0,03)^{*}$ & 5,83 & 88,04 & $(2,05)$ & 5,77 & $(0,22)^{*}$ & 6,15 \\
\hline \multicolumn{11}{|c|}{ Classes de rendimento } \\
\hline $\begin{array}{l}\text { Renda } \\
\mathrm{R} \$ 296,00\end{array}$ & 9,95 & $(0,79)$ & 1,21 & $(0,07)^{*}$ & 10,84 & 98,48 & $(2,24)$ & 11,34 & $(0,37)^{*}$ & 10,33 \\
\hline $\begin{array}{lr}\text { Renda > } & \text { que } \\
\mathrm{R} \$ 296,00 & \mathrm{a}\end{array}$ & 10,32 & $(0,74)$ & 1,55 & $(0,10)^{*}$ & 13,06 & 93,13 & $(2,26)$ & 14,13 & $(0,01)^{*}$ & 13,17 \\
\hline $\begin{array}{l}\mathrm{R} \$ 571,00 \\
\text { Renda > que } \\
\mathrm{R} \$ 571,00 \quad \mathrm{a}\end{array}$ & 9,90 & $(0,69)$ & 1,99 & $(0,12)^{*}$ & 16,74 & 86,75 & $(2,36)$ & 17,26 & $(0,82)^{*}$ & 16,59 \\
\hline $\begin{array}{l}\mathrm{R} \$ 1.089,00 \\
\text { Renda > que } \\
\mathrm{R} \$ 1.089,00\end{array}$ & 8,99 & $(0,57)$ & 2,55 & $(0,18)^{*}$ & 22,10 & 83,79 & $(2,87)$ & 24,95 & $(0,92)^{*}$ & 22,91 \\
\hline
\end{tabular}

Pode-se verificar que para a ingestão de vitaminas e minerais antioxidantes, o consumo alimentar fora do domićlio foi maior entre os homens (16,2 a 20,1\%), para os moradores da zona urbana (entre 14,8 e 17,3\%), entre os adultos (16,1 a 20,1\%), e para indivíduos com renda superior a $\mathrm{R} \$ 1.089,00$ (entre 17,7 e 22,9\%). Já entre as regiões geográficas, a ingestão de vitamina $\mathrm{A}$ e $\mathrm{C}$ fora do domicílio foram maiores na região Norte $(15,9 \%$ e $21,7 \%)$, para a vitamina $E$ na região Sul $(16,0 \%)$, para o zinco nas regiões Sudeste e Sul (16,2\% em ambos), para o selênio na região Sul
$(16,5 \%)$, e para o cobre e manganês foi maior na região Sudeste $(15,2 \%$ e $15,3 \%)$. A ingestão de antioxidantes fora do domicilio se revelou inferior para os moradores da região Nordeste, para os idosos e para os indivíduos com renda até $\mathrm{R} \$ 296$, com exceção da vitamina $\mathrm{C}$, cuja contribuição foi inferior para o grupo com renda que integra o intervalo de $\mathrm{R} \$ 571$ a $\mathrm{R} \$ 1.089$.

Em estudo semelhante a este, comparando as características da alimentação dentro e fora do domicílio, Hoffmann (2013) ${ }^{[24]}$ concluiu que a renda per 
capita média das pessoas que eventualmente comem fora do domicílio é $45 \%$ superior ao rendimento do grupo que não o faz. Acredita-se que as pessoas com renda mais elevada são aquelas que provavelmente realizam as refeições fora do domicílio devido rotina de trabalho, ou até devido a comodidade proporcionada.

Tabela 3. Estimativa da ingestão de minerais antioxidantes cobre e manganês da população brasileira no domicilio e fora do domicílio de acordo com diferentes variáveis. Brasil 2008-2009

\begin{tabular}{|c|c|c|c|c|c|c|c|c|c|c|}
\hline & \multicolumn{5}{|c|}{ Cobre (mg/dia) } & \multicolumn{5}{|c|}{ Manganês (mg/dia) } \\
\hline & \multicolumn{2}{|c|}{ D } & \multicolumn{2}{|c|}{ FD } & \multirow{2}{*}{$\begin{array}{l}\text { \%FD } \\
11,19\end{array}$} & \multicolumn{2}{|c|}{ D } & \multicolumn{2}{|c|}{ FD } & \multirow{2}{*}{$\begin{array}{l}\text { \%FD } \\
10,88\end{array}$} \\
\hline População total & 1,19 & $(0,15)$ & 0,15 & $(0,23)^{*}$ & & 2,62 & $(0,35)$ & 0,32 & $(0,05)^{*}$ & \\
\hline \multicolumn{11}{|l|}{ Sexo } \\
\hline Sexo Masculino & 1,25 & $(0,18)$ & 0,24 & $(0,02)^{*}$ & 16,38 & 2,74 & $(0,38)$ & 0,53 & $(0,07)^{*}$ & 16,21 \\
\hline Sexo Feminino & 1,09 & $(0,14)$ & 0,15 & $(0,01)^{*}$ & 12,10 & 2,31 & $(0,33)$ & 0,33 & $(0,04)^{*}$ & 12,50 \\
\hline \multicolumn{11}{|l|}{ Situação do domicílio } \\
\hline Urbano & 1,15 & $(0,14)$ & 0,20 & $(0,02)^{*}$ & 14,81 & 2,46 & $(0,33)$ & 0,44 & $(0,06)^{*}$ & 15,17 \\
\hline Rural & 1,38 & $(0,23)$ & 0,11 & $(0,01)^{*}$ & 7,38 & 2,91 & $(0,45)$ & 0,26 & $(0,03)^{*}$ & 8,20 \\
\hline \multicolumn{11}{|l|}{ Região } \\
\hline Norte & 1,06 & $(0,14)$ & 0,18 & $(0,01)^{*}$ & 14,52 & 2,28 & $(0,30)$ & 0,39 & $(0,05)^{*}$ & 14,61 \\
\hline Nordeste & 1,21 & $(0,17)$ & 0,16 & $(0,01)^{*}$ & 11,68 & 2,57 & $(0,41)$ & 0,34 & $(0,04)^{*}$ & 11,68 \\
\hline Sudeste & 1,12 & $(0,13)$ & 0,20 & $(0,02)^{*}$ & 15,15 & 2,49 & $(0,33)$ & 0,45 & $(0,06)^{*}$ & 15,31 \\
\hline Sul & 1,38 & $(0,25)$ & 0,22 & $(0,03)^{*}$ & 13,75 & 2,70 & $(0,36)$ & 0,45 & $(0,06)^{*}$ & 14,29 \\
\hline Centro-Oeste & 1,11 & $(0,12)$ & 0,19 & $(0,02)^{*}$ & 14,62 & 2,48 & $(0,32)$ & 0,44 & $(0,07)^{*}$ & 15,07 \\
\hline \multicolumn{11}{|l|}{ Estágio de vida } \\
\hline Adolescentes & 1,17 & $(0,16)$ & 0,15 & $(0,01)^{*}$ & 11,36 & 2,62 & $(0,37)$ & 0,36 & $(0,06)^{*}$ & 12,08 \\
\hline Adultos & 1,16 & $(0,17)$ & 0,23 & $(0,02)^{*}$ & 16,55 & 2,49 & $(0,35)$ & 0,48 & $(0,07)^{*}$ & 16,16 \\
\hline Idosos & 1,27 & $(0,11)$ & 0,06 & $(0,00)^{*}$ & 4,51 & 2,59 & $(0,35)$ & 0,14 & $(0,02)^{*}$ & 5,13 \\
\hline \multicolumn{11}{|l|}{ Classes de rendimento } \\
\hline Renda até $\mathrm{R} \$ 296,00$ & 1,21 & $(0,20)$ & 0,13 & $(0,01)^{*}$ & 9,70 & 2,63 & $(0,39)$ & 0,29 & $(0,04)^{*}$ & 9,93 \\
\hline $\begin{array}{l}\text { Renda > que } \mathrm{R} \$ 296,00 \quad \mathrm{a} \\
\mathrm{R} \$ 571,00\end{array}$ & 1,20 & $(0,20)$ & 0,17 & $(0,02)^{*}$ & 12,41 & 2,65 & $(0,37)$ & 0,36 & $(0,04)^{*}$ & 11,96 \\
\hline $\begin{array}{l}\text { Renda > que } \mathrm{R} \$ 571,00 \text { a } \\
\mathrm{R} \$ 1.089,00\end{array}$ & 1,15 & $(0,17)$ & 0,21 & $(0,02)^{*}$ & 15,44 & 2,50 & $(0,34)$ & 0,46 & $(0,06)^{*}$ & 15,54 \\
\hline Renda $>$ que $\mathrm{R} \$ 1.089,00$ & 1,13 & $(0,04)$ & 0,29 & $(0,02)^{*}$ & 20,42 & 2,26 & $(0,30)$ & 0,61 & $(0,08)^{*}$ & 21,25 \\
\hline
\end{tabular}

A população adulta se destacou no tocante à ingestão de nutrientes antioxidantes fora do domicílio, em comparação aos demais estágios de vida, provavelmente pela falta de tempo para preparar suas refeições, visto a inserção destes indivíduos no mercado de trabalho[ ${ }^{[2]}$. Em relação aos idosos que, por sua vez, apresentaram baixa ingestão de nutrientes antioxidantes em comparação aos demais estágios de vida, verifica-se que o consumo alimentar dos mesmos pode ser influenciado por diversos fatores, tais como modificações próprias do envelhecimento (o que muitas vezes pode dificultar a mobilidade), e o fato da escolha alimentar dos mesmos estar relacionada ao preço e a qualidade nutricional dos alimentos ${ }^{[25]}$.
Amancio e Silva (2012)[26] ao analisarem o consumo de carotenoides da população brasileira fora do domicílio, relataram que os moradores da zona urbana têm maior facilidade em adquirir alimentos mais variados, assim como para alimentar-se fora do domicílio, comparando-se aos moradores da zona rural. Isto devido aos locais que comercializam alimentos ou refeições prontas estarem geralmente situados relativamente próximos às suas residências e ou locais de trabalho.

Dados semelhantes foram relatados por Bezerra et al. (2010) [27], que ao avaliarem os gastos com a alimentação fora do domicílio no Brasil, observaram que o percentual de indivíduos com gasto de 
alimentação fora do domicílio diminuiu com a idade e aumentou com a renda em todas as regiões brasileiras. Mais homens reportaram o consumo fora do domicílio, assim como os indivíduos residentes da área urbana. Os grupos de alimentos consumidos com maior frequência fora do domicílio foram bebidas alcoólicas e salgadinhos fritos e assados.

O consumo alimentar fora do domicilio destacou-se nas regiões economicamente mais desenvolvidas. Nesse sentido, Neves, Koifman e Mattos (2006) ${ }^{[28]}$ realizaram um estudo (nas capitais brasileiras) relacionando a mortalidade por câncer de cólon e o consumo alimentar. Os autores observaram que nas capitais das regiões Sul, Sudeste e Centro-oeste houve maior consumo de energia (calorias), enquanto um consumo abaixo da média foi observado nas capitais do Norte e do Nordeste. Além disso, encontraram correlações significativamente positivas entre as taxas de morte por câncer e as variáveis renda e consumo de calorias, óleos e gorduras e carnes.

Ao analisar os grupos de alimentos que mais contribuíram para a ingestão total de vitaminas antioxidantes fora do domicílio, verificou-se que para a ingestão de vitamina A houve maior participação do grupo de verduras $(22,57 \%)$, dos doces $(21,31 \%)$ e das carnes e ovos $(11,95 \%)$, destacando-se a couve $(7,21 \%)$, doces à base de leite $(12,73 \%)$, ovo de galinha $(5,05 \%)$ e fígado bovino (4,48\%), os quais são reconhecidamente os principais grupos fontes $\mathrm{da}$ vitamina (Tabela 4). Ainda, é interessante mencionar que com relação à participação da alimentação fora do domicílio, o grupo das verduras apresentou importante contribuição. Tureck et al. (2013)[19] ao avaliarem a ingestão total de vitaminas antioxidantes verificaram que os grupos que mais contribuíram para a ingestão de vitamina A foram os grupos das carnes e ovos e dos laticínios.

Tabela 4. Estimativa da ingestão de vitaminas antioxidantes pela população brasileira dentro e fora do domicílio, de acordo com grupos alimentares. Brasil 2008, 2009

\begin{tabular}{|c|c|c|c|c|c|c|c|c|c|c|c|c|c|c|c|c|c|}
\hline \multirow{2}{*}{$\begin{array}{c}\text { Grupos } \\
\text { alimentares }\end{array}$} & \multirow{2}{*}{$\begin{array}{c}\begin{array}{c}\text { Fora do } \\
\text { domicílio }\end{array} \\
\text { Porção } \\
(\mathrm{g} / \mathrm{mL})\end{array}$} & \multirow{2}{*}{$\begin{array}{c}\text { Domicílio } \\
\text { Porção } \\
(\mathrm{g} / \mathrm{mL}) \\
\end{array}$} & \multicolumn{5}{|c|}{ Vitamina A ( $\mu \mathrm{g} / \mathrm{dia})$} & \multicolumn{5}{|c|}{ Vitamina C (mg/dia) } & \multicolumn{5}{|c|}{ Vitamina E (mg/dia) } \\
\hline & & & \multicolumn{2}{|c|}{ FD } & \multicolumn{2}{|c|}{ D } & \multirow[t]{2}{*}{$\% \mathrm{FD}$} & \multicolumn{2}{|c|}{ FD } & \multicolumn{2}{|c|}{$\mathbf{D}$} & \multirow{2}{*}{$\begin{array}{r}\mathbf{\%} \mathbf{F D} \\
1,23\end{array}$} & \multicolumn{2}{|c|}{ FD } & \multicolumn{2}{|c|}{ D } & \multirow{2}{*}{$\begin{array}{l}\text { \%FD } \\
47,06\end{array}$} \\
\hline Cereais & 22,650 & 169,350 & 0,12 & $(0,03)$ & 3,20 & $(1,72)$ & & 0,11 & $(0,00)$ & 0,75 & $(0,00)$ & & 0,24 & $(0,00)$ & 1,79 & $(0,00)$ & \\
\hline Leguminosas & 23,410 & 175,490 & 0,22 & $(0,09)$ & 2,37 & $(1,31)$ & 0,79 & 0,02 & $(0,01)$ & 0,29 & $(0,14)$ & 0,22 & 0,00 & $(0,00)$ & 0,04 & $(0,00)$ & 0,00 \\
\hline Verduras & 6,029 & 34,000 & 6,27 & $(2,79)$ & 30,66 & $(13,66)$ & 22,57 & 1,74 & $(0,09)$ & 9,23 & $(0,48)$ & 19,46 & 0,07 & $(0,04)$ & 0,35 & $(0,18)$ & 13,73 \\
\hline $\begin{array}{l}\text { Raízes } \mathrm{e} \\
\text { tubérculos }\end{array}$ & 4,020 & 20,980 & 1,69 & $(0,08)$ & 27,20 & $(1,00)$ & 6,08 & 0,47 & $(0,28)$ & 2,83 & $(1,54)$ & 5,26 & 0,01 & $(0,00)$ & 0,08 & $(0,04)$ & 1,96 \\
\hline Frutas & 10,600 & 75,500 & 1,93 & $(0,04)$ & 25,77 & $(13,79)$ & 6,95 & 3,28 & $(0,04)$ & 33,57 & $(3,56)$ & 36,69 & 0,03 & $(0,00)$ & 0,21 & $(0,01)$ & 5,88 \\
\hline Oleaginosas & 0,009 & 0,391 & 0,00 & $(0,00)$ & 0,00 & $(0,00)$ & 0,00 & 0,00 & $(0,00)$ & 0,01 & $(0,00)$ & 0,00 & 0,00 & $(0,00)$ & 0,02 & $(0,00)$ & 0,00 \\
\hline $\begin{array}{l}\text { Farinha, } \\
\text { massas, } \\
\text { panificados e } \\
\text { biscoitos }\end{array}$ & 10,980 & 122,420 & 1,33 & $(0,27)$ & 14,28 & $(1,55)$ & 4,79 & 0,11 & $(0,01)$ & 1,18 & $(0,16)$ & 1,23 & 0,07 & $(0,00)$ & 0,49 & $(0,06)$ & 13,73 \\
\hline $\begin{array}{l}\text { Carnes } \\
\text { ovos }\end{array}$ & 14,580 & 151,020 & 3,32 & $(0,51)$ & 80,93 & $(15,87)$ & 11,95 & 0,12 & $(0,00)$ & 1,26 & $(0,06)$ & 1,34 & 0,04 & $(0,00)$ & 0,58 & $(0,00)$ & 7,84 \\
\hline Laticínios & 6,350 & 68,150 & 2,03 & $(0,49)$ & 32,29 & $(7,30)$ & 7,31 & 0,18 & $(0,03)$ & 1,69 & $(0,32)$ & 2,01 & 0,00 & $(0,00)$ & 0,06 & $(0,00)$ & 0,00 \\
\hline Doces & 13,330 & 7,370 & 5,92 & $(0,77)$ & 2,55 & $(0,98)$ & 21,31 & 0,60 & $(0,13)$ & 0,39 & $(0,35)$ & 6,71 & 0,03 & $(0,00)$ & 0,06 & $(1,96)$ & 5,88 \\
\hline $\begin{array}{l}\text { Óleos } \\
\text { gorduras }\end{array}$ & 0,070 & 6,530 & 0,16 & $(0,00)$ & 14,97 & $(0,46)$ & 0,58 & 0,00 & $(0,00)$ & 0,00 & $(0,00)$ & 0,00 & 0,00 & $(0,00)$ & 0,71 & $(0,56)$ & 0,00 \\
\hline Bebidas & 74,060 & 470,340 & 0,63 & $(0,14)$ & 7,40 & $(2,28)$ & 2,27 & 2,12 & $(0,69)$ & 31,23 & $(10,50)$ & 23,71 & 0,00 & $(0,00)$ & 0,12 & $(0,00)$ & 0,00 \\
\hline $\begin{array}{l}\text { Salgados e } \\
\text { sanduíches }\end{array}$ & 4,220 & 22,480 & 3,06 & $(2,55)$ & 14,28 & $(9,30)$ & 11,02 & 0,15 & $(0,11)$ & 0,69 & $(0,42)$ & 1,68 & 0,02 & $(0,00)$ & 0,17 & $(0,04)$ & 3,92 \\
\hline $\begin{array}{l}\text { Sopas } \\
\text { caldos }\end{array}$ & 2,890 & 47,410 & 1,10 & $(0,45)$ & 18,06 & $(7,41)$ & 3,96 & 0,04 & $(0,05)$ & 0,75 & $(0,84)$ & 0,45 & 0,00 & $(0,00)$ & 0,03 & $(0,00)$ & 0,00 \\
\hline $\begin{array}{l}\text { Molhos e } \\
\text { condimentos }\end{array}$ & 0,010 & 0,490 & 0,00 & $(0,00)$ & 0,12 & $(0,04)$ & 0,00 & 0,00 & $(0,00)$ & 0,05 & $(0,00)$ & 0,00 & 0,00 & $(0,00)$ & 0,00 & $(0,00)$ & 0,00 \\
\hline
\end{tabular}

Em relação à ingestão total de vitamina $\mathrm{C}$ (Tabela 4), os grupos com maior contribuição (alimentação fora do domicilio) foram as frutas $(36,69 \%)$, com destaque para as cítricas como laranja
$(20,04 \%)$ e tangerina (3,15\%), que são boas fontes desta vitamina, e o mamão $(4,38 \%)$, as bebidas $(23,71 \%)$, notadamente o suco de laranja $(20,08 \%)$ e as verduras $(19,46 \%)$, principalmente couve $(8,33 \%)$ e tomate 
(6,24\%). Os mesmos resultados foram encontrados por Tureck et al. (2013) ${ }^{[19]}$, considerando-se a ingestão total de antioxidantes.

Já em relação à ingestão total de vitamina $\mathrm{E}$ (Tabela 4), os grupos que mais contribuíram para o consumo fora do domicílio foram o dos cereais $(47,06 \%)$, verduras $(13,73 \%)$ e farinhas, massas, panificados e biscoitos $(13,73 \%)$, destacando-se nestes grupos o arroz $(37,92 \%)$, couve $(6,24 \%)$ e farofa (5,64\%). Tureck et al. (2013) ${ }^{[19]}$ também observaram maior contribuição do grupo dos cereais para a ingestão total desta vitamina.
Para a ingestão de zinco e selênio (Tabela 5) os grupos que mais contribuíram foram o das carnes e ovos $(50,76 \%)$, leguminosas $(15,91 \%)$ e doces $(10,61 \%)$, destacando-se a carne bovina (38,81\%), feijão $(14,81 \%)$ e chocolate $(4,34 \%)$. Ainda no tocante à contribuição de selênio, o grupo de maior destaque foi representado pelas carnes e ovos (40,99\%), principalmente a carne bovina $(24,44 \%)$, boa fonte do mineral, seguido do grupo das farinhas, massas e panificados $(19,21 \%)$ e dos cereais $(14,06 \%)$, destacando-se o macarrão $(13,23 \%)$ e $\operatorname{arroz}(12,30 \%)$.

Tabela 5. Estimativa da ingestão de minerais antioxidantes pela população brasileira dentro e fora do domicílio, de acordo com os grupos alimentares. Brasil 2008, 2009

\begin{tabular}{|c|c|c|c|c|c|c|c|c|c|c|c|c|c|c|c|c|c|c|c|c|}
\hline \multirow{2}{*}{$\begin{array}{c}\text { Grupos } \\
\text { alimentares }\end{array}$} & \multicolumn{5}{|c|}{ Zinco (mg/dia) } & \multicolumn{5}{|c|}{ Selênio $(\mu \mathrm{g} / \mathrm{dia})$} & \multicolumn{5}{|c|}{ Cobre $(\mathrm{mg} / \mathrm{dia})$} & \multicolumn{5}{|c|}{ Manganês (mg/dia) } \\
\hline & \multicolumn{2}{|c|}{ FD } & \multicolumn{2}{|c|}{ D } & \multirow{2}{*}{$\begin{array}{c}\text { \%FD } \\
9,09\end{array}$} & \multicolumn{2}{|c|}{ FD } & \multicolumn{2}{|c|}{ D } & \multirow{2}{*}{$\begin{array}{l}\% \text { FD } \\
14,06\end{array}$} & \multicolumn{2}{|c|}{ FD } & \multicolumn{2}{|c|}{ D } & \multirow{2}{*}{$\begin{array}{c}\text { \%FD } \\
0,00\end{array}$} & \multicolumn{2}{|c|}{ FD } & \multicolumn{2}{|c|}{ D } & \multirow{2}{*}{$\begin{array}{l}\% \text { FD } \\
26,67\end{array}$} \\
\hline Cereais & 0,12 & $(0,00)$ & 1,08 & $(0,00)$ & & 1,42 & $(0,33)$ & 10,4 & $(1,64)$ & & 0,00 & $(0,00)$ & 0,10 & $(0,02)$ & & 0,08 & $(0,01)$ & 0,64 & $(0,08)$ & \\
\hline Leguminosas & 0,21 & $(0,07)$ & 1,58 & $(0,54)$ & 15,91 & 0,33 & $(0,01)$ & 2,14 & $(0,17)$ & 3,27 & 0,10 & $(0,00)$ & 0,35 & $(0,01)$ & 45,45 & 0,09 & $(0,01)$ & 0,71 & $(0,11)$ & 30,00 \\
\hline Verduras & 0,01 & $(0,00)$ & 0,06 & $(0,00)$ & 0,76 & 0,03 & $(0,00)$ & 0,32 & $(0,09)$ & 0,30 & 0,00 & $(0,00)$ & 0,02 & $(0,00)$ & 0,00 & 0,01 . & $(0,00)$ & 0,07 & $(0,00)$ & 3,33 \\
\hline Raízes e & & & & & & & & & & & & & & & & & & & & \\
\hline tubérculos & 0,00 & $(0,00)$ & 0,05 & $(0,00)$ & 0,00 & 0,02 & $(0,00)$ & 0,11 & $(0,03)$ & 0,20 & 0,00 & $(0,00)$ & 0,02 & $(0,01)$ & 0,00 & 0,00 & $(0,00)$ & 0,03 & $(0,02)$ & 0,00 \\
\hline Frutas & 0,00 & $(0,00)$ & 0,09 & $(0,01)$ & 0,00 & 0,05 & $(0,00)$ & 0,42 & $(0,03)$ & 0,50 & 0,00 & $(0,00)$ & 0,08 & $(0,06)$ & 0,00 & 0,01 & $(0,00)$ & 0,11 & $(0,01)$ & 3,33 \\
\hline Oleaginosas & 0,00 & $(0,00)$ & 0,01 & $(0,00)$ & 0,00 & 0,02 & $(0,00)$ & 1,09 & $(0,29)$ & 0,20 & 0,00 & $(0,00)$ & 0,00 & $(0,00)$ & 0,00 & 0,00 & $(0,00)$ & 0,00 & $(0,00)$ & 0,00 \\
\hline Farinhas...** & 0,07 & $(0,00)$ & 0,85 & $(0,03)$ & 5,30 & 1,94 & $(0,25)$ & 25,67 & $(1,40)$ & 19,21 & 0,00 & $(0,00)$ & 0,16 & $(0,02)$ & 9,09 & 0,05 & $(0,00)$ & 0,55 & $(0,03)$ & 16,67 \\
\hline Carnes $\mathrm{e}$ & & & & & & & & & & & & & & & & & & & & \\
\hline ovos & 0,67 & $(0,00)$ & 5,16 & $(0,01)$ & 50,76 & 4,14 & $(0,05)$ & 47,93 & $(1,01)$ & 40,99 & 0,00 & $(0,00)$ & 0,16 & $(0,00)$ & 9,09 & 0,00 & $(0,00)$ & 0,05 & $(0,00)$ & 0,00 \\
\hline Laticínios & 0,02 & $(0,00)$ & 0,40 & $(0,02)$ & 1,52 & 0,15 & $(0,07)$ & 2,29 & $(0,89)$ & 1,49 & 0,00 & $(0,00)$ & 0,01 & $(0,00)$ & 0,00 & 0,00 & $(0,00)$ & 0,00 & $(0,00)$ & 0,00 \\
\hline $\begin{array}{l}\text { Doces } \\
\text { Óleos }\end{array}$ & 0,14 & $(0,07)$ & 0,05 & $(0,00)$ & 10,61 & 1,04 & $(0,00)$ & 0,58 & $(0,00)$ & 10,30 & 0,00 & $(0,00)$ & 0,02 & $(0,00)$ & 18,18 & 0,02 & $(0,00$ & 0,03 & $(0,00)$ & 6,67 \\
\hline gorduras & 0,00 & $(0,00)$ & 0,01 & $(0,00)$ & 0,00 & 0,01 & $(0,00)$ & 1,12 & $(0,00)$ & 0,10 & 0,00 & $(0,00)$ & 0,00 & $(0,00)$ & 0,00 & 0,00 & $(0,00)$ & 0,00 & $(0,00)$ & 0,00 \\
\hline Bebidas & 0,04 & $(0,01)$ & 0,6 & $(0,27)$ & 3,03 & 0,29 & $(0,09)$ & 1,06 & $(0,03)$ & 2,87 & 0,00 & $(0,01)$ & 0,21 & $(0,15)$ & 18,18 & 0,03 & $(0,00)$ & 0,30 & $(0,12)$ & 10,00 \\
\hline $\begin{array}{l}\text { Salgados e } \\
\text { sanduíches } \\
\text { Sopas e }\end{array}$ & 0,04 & $(0,00)$ & 0,27 & $(0,04)$ & 3,03 & 0,59 & $(0,11)$ & 3,07 & $(0,70)$ & 5,84 & 0,00 & $(0,00)$ & 0,03 & $(0,00)$ & 0,00 & 0,01 & $(0,00)$ & 0,06 & $(0,00)$ & 0,35 \\
\hline $\begin{array}{l}\text { caldos } \\
\text { Molhos }\end{array}$ & 0,00 & $(0,00)$ & 0,15 & $(0,09)$ & 0,00 & 0,07 & $(0,03)$ & 1,25 & $(0,51)$ & 0,69 & 0,00 & $(0,00)$ & 0,02 & $(0,00)$ & 0,00 & 0,00 & $(0,00)$ & 0,04 & $(0,00)$ & 0,00 \\
\hline condimentos & 0,00 & $(0,00)$ & 0,00 & $(0,00)$ & 0,00 & 0,00 & $(0,00)$ & 0,03 & $(0,00)$ & 0,00 & 0,00 & $(0,00)$ & 0,00 & $(0,00)$ & 0,00 & 0,00 & $(0,00)$ & 0,00 & $(0,00)$ & 0,00 \\
\hline
\end{tabular}

Em relação ao cobre (Tabela 5), os grupos que mais contribuíram para a ingestão deste antioxidante fora do domicilio foram leguminosas $(45,45 \%)$, doces $(18,18 \%)$ e bebidas $(18,18 \%)$, destacando-se o feijão $(30,63 \%)$, chocolate $(9,40 \%)$ e café $(7,29 \%)$. E, para a ingestão de manganês fora do domicilio os grupos que mais contribuíram foram o das leguminosas $(30 \%)$, seguido dos cereais $(26,67 \%)$, e farinhas, massas e panificados $(16,67 \%)$, sendo que ambos representam boas fontes do mineral, destacando-se feijão $(28,17 \%)$, arroz $(20,47 \%)$ e macarrão (5,31\%). Estes resultados também são semelhantes aos observados por Tureck $e t$ al. (2013) [19], que mostraram que as leguminosas representaram a maior contribuição para a ingestão total de cobre e manganês.

Avaliando-se a distribuição regional e socioeconômica da disponibilidade domiciliar de alimentos no Brasil e sua evolução, Levy-Costa et al. (2005) ${ }^{[29]}$ e Levy et al. (2011) ${ }^{[30]}$ observaram que o teor proteico das dietas mostrou-se adequado em todas as regiões e classes de rendimentos. Especialmente nas áreas urbanas e nas regiões economicamente mais desenvolvidas, os autores verificaram o consumo elevado de gorduras saturadas, bem como o declínio no consumo de alimentos básicos, como arroz e feijão, aumento no consumo de produtos industrializados, 
como biscoitos e refrigerantes, persistência no consumo de frutas e hortaliças e aumento de gorduras na dieta.

Duarte, Almeida e Martins (2013) ${ }^{[31]}$ ao avaliar o consumo alimentar fora do domicílio de universitários de uma instituição privada (parcela não representativa da população brasileira), verificaram que muitos universitários passam o dia fora de casa. Ainda, as autoras identificaram que $84 \%$ dos entrevistados adotavam o referido hábito. A refeição de maior consumo foi o almoço e o local mais procurado foi "restaurante por quilo". Os alimentos mais consumidos foram leite/derivados, frutas, doces, salgados, biscoitos, fast foods e refrigerantes.

Hoffmann (2013) ${ }^{[24]}$ comparando o hábito de comer fora do domicílio e o Índice de Massa Corporal (IMC) da população brasileira, concluiu que não se pode considerar que a alimentação fora do domicilio seja de pior qualidade, pois embora o teor de açúcar seja maior, o teor de lipídeos foi inferior e o teor de vitamina $\mathrm{C}$ superior ao da alimentação ingerida no domićlilio. $\mathrm{O}$ autor ressalta que no Brasil não é correto identificar a alimentação fora do domicilio como ingestão de fast food. Em relação ao IMC, não foram identificadas correlações entre o hábito de comer fora do domicílio e a ocorrência de sobrepeso e obesidade.

Destaca-se que ao analisar a alimentação fora do domićlio é preciso considerar que não se refere apenas à alimentação realizada em bares, lanchonetes e restaurantes, mas inclui a alimentação realizada no local de trabalho e nas escolas, locais onde nem sempre as recomendações nutricionais são atingidas. Geraldo, Bandoni e Jaime (2008) ${ }^{[32]}$ analisando a alimentação do Programa de Alimentação do Trabalhador - PAT, verificaram que a oferta de energia, proteínas $\mathrm{e}$ colesterol eram superiores às recomendadas, e a oferta de frutas e hortaliças superava à recomendação. Já no que diz respeito à alimentação escolar, Silva e Gregório (2008) ${ }^{[33]}$ e Flávio (2008) ${ }^{[34]}$, observaram inadequação de energia, macro e micronutrientes nos cardápios escolares oferecidos.

De maneira geral, verifica-se que as refeições realizadas fora do domicílio crescem cada vez mais, especialmente na zona urbana, nas regiões mais economicamente desenvolvidas e para a população adulta. Com isso, as pessoas têm optado por praticidade e buscam ambientes que disponibilizem refeições rápidas, mas que nem sempre são saudáveis. Neste sentido, a oferta de alimentos saudáveis pelos serviços de alimentação, principalmente daqueles reconhecidos como fontes de nutrientes antioxidantes, bem como a reeducação alimentar das pessoas é fundamental para a promoção da qualidade de vida e prevenção de doenças, uma vez que os nutrientes antioxidantes proporcionam os efeitos benéficos ao atenuar o estresse oxidativo.

Ainda, acredita-se que a baixa contribuição da alimentação fora do domicílio para a ingestão de vitaminas e minerais antioxidantes se deve as escolhas alimentares dos indivíduos, visto que optam por alimentos calóricos e com alto teor de gordura e açúcar, em detrimento dos alimentos fontes de nutrientes antioxidantes.

\section{CONCLUSÕES}

Os resultados obtidos por meio deste estudo demonstraram que o consumo alimentar fora do domicílio predominou entre os homens, sendo mais frequente entre a população urbana e adulta. A região nordeste foi a que apresentou menor consumo fora do domicilio.

Embora o consumo alimentar fora do domićlio venha crescendo ao longo dos anos, sua participação na ingestão de vitaminas e minerais antioxidantes foi classificada como baixa (apenas 10\%). No entanto, apesar de ser bem reconhecido o consumo de alimentos ricos em calorias e pobres em vitaminas e minerais fora do domicílio, verificou-se que a participação de frutas, legumes e verduras, assim como cereais e leguminosas foi importante para a ingestão de vitaminas e minerais antioxidantes.

Assim sendo, a oferta de refeições saudáveis com variedade e qualidade pelos serviços de alimentação, com o incentivo ao consumo de frutas, hortaliças, cereais, leguminosas e oleaginosas podem ser favoráveis para a ingestão de nutrientes antioxidantes.

Ainda, destaca-se a necessidade de fortalecimento dos programas governamentais que servem alimentação para trabalhadores e escolares, de maneira a viabilizar e garantir a adequação nas recomendações nutricionais estabelecidas. Ademais, salienta-se a importância de ações de educação 
Alimentação fora do domicilio e ingestão de antioxidantes. Oliveira, Tureck e Koehnlein

alimentar e nutricional para o alcance da segurança alimentar e nutricional.

\section{REFERÊNCIAS}

[1] Batista Filho M, Souza AI, Miglioli TC, Santos MC. Anemia e obesidade: um paradoxo da transição nutricional brasileira. Cad. Saúde Pública. 2008;24(supl. 2):s247-57.

[2] Moratoya EE, Carvalhães GC, Wander AE, Almeida LMMC. Mudanças no padrão de consumo alimentar no Brasil e no mundo. Revista de Política Agrícola. 2013;22(1):72-84.

[3] Bielemann RM, Motta JVS, Minten GC, Horta BL, Gigante DP. Consumo de alimentos ultraprocessados e impacto na dieta de adultos jovens. Rev. Saúde Pública. 2015;49(28):1-10.

[4] Bezerra IN, Souza AM, Pereira RA, Sichieri R. Consumo de alimentos fora do domicílio no Brasil. Rev. Saúde Pública. 2013;47(1Supl):200S-11S.

[5] Claro RM, Baraldi LG, Martins APB, Bandoni DH, Levy RB. Evolução das despesas com alimentação fora do domicilio e influência da renda no Brasil, 2002/2003 a 2008/2009. Cad. Saúde Pública. 2014,30(7):1418-1426.

[6] Instituto Brasileiro de Geografia e Estatística. Pesquisa de Orçamentos Familiares 2008-2009: análise do consumo alimentar pessoal no Brasil. Rio de Janeiro: IBGE; 2011.

[7] Catania AS, Barros CR, Ferreira SR. Vitaminas e minerais com propriedades antioxidantes e risco cardiometabólico: controvérsias e perspectivas. Arq. Bras. Endocrinol. Metab. 2009;53(5):550-9.

[8] Gottlieb MGV, Morassutti AL, Cruz IBM. Transição epidemiológica, estresse oxidativo e doenças crônicas não transmissíveis sob uma perspectiva evolutiva. Sci. Med. 2011;21(2):69-80.

[9] Barreiros ALBS, David JM, David JP. Estresse oxidativo: relação entre geração de espécies reativas e defesa do organismo. Quim. Nova. 2006;29(1):113-23.

[10] Ambrósio CLB, Campos FACS, Faro ZP. Carotenóides como alternativa contra a hipovitaminose A. Rev. Nutr. 2006;19,(2):233-43.

[11] Boni A, Pugliese C, Cláudio CC, Patin RV, Oliveira FLC. Vitaminas antioxidantes e prevenção da arteriosclerose na infância. Rev. Paul. Pediatr. 2010;28(4):373-80.

[12] Gordon MH. Significance of Dietary Antioxidants for Health. Int. J. Mol. Sci. 2012;13(1):173-9.

[13] Honarbakhsh S, Schachter M. Vitamin sand cardiovascular disease. Br. J. Nutr. 2009;101(8):1113-31.
[14] Mahan LK, Escott-Stump SF. Krause: Alimentos, Nutrição e Dietoterapia. 12.ed. Rio de Janeiro: Florescer; 2010.

[15] Wannamethee SG, Lowe GD, Rumley A, Bruckdorfer KR, Whincup PH. Associations of vitamin C status, fruit and vegetable intakes, and markers of inflammation and hemostasis. Am. J. Clin. Nutr. 2006;83(3):567-74.

[16] Aranha FQ, Barros ZF, Moura LSA, Gonçalves MCR, Barros JC, Metri JC, et al. O papel da Vitamina C sobre as alterações orgânicas no idoso. Rev. Nutr. 2000;13(2):89-97.

[17] Mafra D, Cozzolino SMF. Importância do zinco na nutrição humana. Rev. Nutr. 2004;17(1):79-87.

[18] Volp ACP, Bressan J, Hermsdorff HHM, Zulet MA, Martinez JA. Efeitos antioxidantes do selênio e seu elo com a inflamação e síndrome metabólica. Rev. Nutr. 2010;23(4):581-90.

[19] Tureck C, Gesser Correa V, Peralta RM, Koehnlein EA. Estimativa do consumo de vitaminas e minerais antioxidantes da dieta brasileira. Nutr. Clín. Diet. Hosp. 2013;33(3):30-8.

[20] Nepa. Unicamp. Tabela brasileira de composição de alimentos: TACO. 4ed. Campinas: Nepa-Unicamp; 2011.

[21] Philippi ST. Tabela de Composição de Alimentos: suporte para decisão nutricional. 3.ed. Barueri: Manole; 2012.

[22] Instituto Brasileiro de Geografia e Estatística. Pesquisa de Orçamentos Familiares 2008-2009: tabelas de composição nutricional dos alimentos consumidos no Brasil. Rio de Janeiro: IBGE; 2011.

[23] U.S. Department of Agriculture, Agricultural Research Service. USDA Nutrient Database for Standard Reference, Release 26 online. Nutrient Data Laboratory Home Page [Internet]. Washington, 2015 [acesso em 10 jun 2015]. Disponível em: http://ndb.nal.usda.gov

[24] Hoffmann F. Comparando a alimentação dentro e fora do domicílio, no Brasil, em 2008-2009. Segur. Aliment. Nutr. 2013;20(1):1-12.

[25] Almeida IC, Guimarães GF, Rezende DC. Hábitos alimentares da população idosa: padrões de compra e consumo. Gestão Contemporânea. 2010;7(8):63-92.

[26] Amancio RD, Silva MV. Consumo de carotenoides no Brasil: a contribuição da alimentação fora do domicílio. Segur. Aliment. Nutr. 2012;19(2):130-141.

[27] Bezerra IN, Sichieri R. Características e gastos com alimentação fora do domicílio no Brasil. Rev. Saúde Pública. 2010;44(2):221-9.

[28] Neves PJ, Koifman RJ, Mattos IE. Mortalidade por câncer de cólon e reto e consumo alimentar em capitais brasileiras selecionadas. Rev. Bras. Epidemiol. 2006;9(1):112-20. 
[29] Levy-Costa RB, Sichieri R, Pontes NS, Monteiro CA. Disponibilidade domiciliar de alimentos no Brasil: distribuição e evolução (1974-2003). Rev. Saúde Pública. 2005;39(4):530-40.

[30] Levy RB, Claro RM, Mondini L, Sichieri R, Monteiro CA. Distribuição regional e socioeconômica da disponibilidade domiciliar de alimentos no Brasil em 2008-2009. Rev. Saúde Pública. 2012,46(1):6-15.

[31] Duarte FM, Almeida SDS, Martins KA. Alimentação fora do domicílio de universitários de alguns cursos da área da saúde de uma instituição privada. O mundo da Saúde. 2013;37(3):288-98.

[32] Geraldo APG, Bandoni DH, Jaime PC. Aspectos dietéticos das refeições oferecidas por empresas participantes do Programa de Alimentação do Trabalhador na Cidade de São Paulo, Brasil. Rev. Panam. Salud. Publica. 2008;23(1):19-25.

[33] Silva MMDC, Gregório EL. Avaliação da composição nutricional dos cardápios da alimentação escolar das escolas da rede municipal de Taquaraçu de Minas - MG. HU Revista. 2012;37(3):387-94.

[34] Flávio EF, Piccolo B, Cirillo MA, Ribeiro AH. Avaliação da alimentação escolar oferecida aos alunos do ensino fundamental das escolas municipais de Lavras, MG. Ciênc. Agrotec. 2008;32(6):1879-

87. 